\title{
Nadjaf, grand site de pèlerinage chiite pour les vivants et... pour les morts
}

\section{Gérard-François Dumont}

\section{OpenEdition}

\section{Journals}

Édition électronique

URL : https://journals.openedition.org/geohist/507

DOI : 10.4000/geohist.507

ISSN : 2264-2617

Éditeur

Association française de la Revue de géographie historique

Référence électronique

Gérard-François Dumont, « Nadjaf, grand site de pèlerinage chiite pour les vivants et... pour les morts », Revue de géographie historique [En ligne], 16| 2020, mis en ligne le 20 mai 2020, consulté le 12 juin 2021. URL : http://journals.openedition.org/geohist/507 ; DOI : https://doi.org/10.4000/geohist. 507

Ce document a été généré automatiquement le 12 juin 2021.

Ce(tte) œuvre est mise à disposition selon les termes de la Licence Creative Commons Attribution Pas d'Utilisation Commerciale - Pas de Modification 4.0 International. 


\title{
Nadjaf, grand site de pèlerinage chiite pour les vivants et... pour les morts
}

\author{
Gérard-François Dumont
}

1 L'insuffisante connaissance de la diversité religieuse conduit trop souvent à omettre les variétés de l'islam et ses divers lieux de pèlerinage en Asie comme en Afrique. Parmi ces derniers, Nadjaf, ville située à $160 \mathrm{~km}$ au sud de la capitale irakienne Bagdad, tient une place très importante comme ville de pèlerinage et lieu de départ de la principale procession chiite annuelle. En outre, sa fonction de pèlerinage dévotionnel se complète doublement, d'une part, par ce qu'il convient d'appeler de longs «pèlerinages théologiques » et, d'autre part, par un dernier pèlerinage post mortem de la dépouille des croyants.

2 Commençons par la première fonction de pèlerinage dévotionnel, a priori, assez banale puisqu'elle existe dans de nombreux lieux dans le monde, mais dont il convient de comprendre l'importance puisque Nadjaf est le troisième lieu saint de l'islam chiite, après La Mecque et Médine et devant Kerbala. En outre, sa durée, depuis près d'un millénaire, retient l'attention, même si le pèlerinage dévotionnel s'est effectué de façon discontinue en raison des vicissitudes de l'histoire.

\section{Un millénaire de pèlerinage dévotionnel avec des éclipses géopolitiques}

3 La ville de Nadjaf ne s'est pas initialement développée grâce à un pèlerinage dévotionnel comme, par exemple, Rocamadour en France. En effet, avant l'islam, Nadjaf $^{1}$ est déjà un site urbain, sachant que le plus ancien texte y évoquant un peuplement remonte au règne du roi babylonien Nabuchodonosor (626-539 avant J.-C.). $\mathrm{Au}$ fil des siècles, la ville, très appréciée comme colline aérée à une dizaine de kilomètres de la rive droite de l'Euphrate, voit s'y construire des palais, des temples, puis, dès les premiers siècles de la chrétienté, des églises et des monastères. Nadjaf est 
alors citée dans des poésies de la période préislamique, dont celles de la période chrétienne qui dure jusqu'à l'arrivée de l'islam au VII siècle.

La ville est alors à nouveau citée dans des textes qui relatent la conquête islamique de la Mésopotamie, puis n'apparaît plus jusqu'à la découverte en son lieu du tombeau de l'imam Ali, découverte incontournable pour comprendre le troisième rang de cette ville pour les pèlerinages dévotionnels chiites. Mais il est impossible de comprendre l'importance de son pèlerinage dévotionnel sans rappeler le contexte de la genèse du chiisme.

\section{A. La sécession des partisans d'Ali}

Lorsque Mahomet meurt en 632, son entourage nomme comme successeur à la tête des musulmans Abou Bakr, le premier calife (précisément Khalifa rasoul Allah, soit successeur de l'envoyé de Dieu) qui meurt deux ans plus tard. Omar, deuxième calife, désigné comme successeur d'Abou Bakr, meurt assassiné en 644. Il est remplacé par Uthman, troisième calife, à son tour assassiné en 656 . Est alors désigné un quatrième calife, dont certains pensent qu'il aurait dû être le premier calife car il aurait été l'un des premiers, avec Khadija, la première épouse de Mohamed, a avoir cru en la mission religieuse de Mahomet. Certains pensent, sans avoir de preuves formelles, qu'il aurait en outre été désigné par Mahomet pour lui succéder, donc le seul à prétendre légitimement à la direction des musulmans après Mahomet. D'autres considèrent que, en 632, l'entourage de Mahomet l'avait jugé trop jeune. Ce quatrième calife est Ali ibn Abî-Tâlib, né vers l'année 600 à La Mecque, cousin de Mahomet et son gendre puisqu'il est marié avec la première fille de Mahomet, Fatima.

Mais une partie des musulmans n'accepte pas l'autorité d'Ali. En particulier Mu'awiyya (602-680), qui gouverne la Syrie, refuse de lui prêter allégeance. La guerre entre différents clans musulmans se déploie. En 656, Ali remporte une victoire militaire à la Bataille du Chameau. Toutefois, considérant ses forces militaires limitées, Ali signe un compromis avec ses adversaires, ce qui n'est pas apprécié par les plus intransigeants qui s'en éloigne en créant la branche islamique des Kharidjites (ou sortants). Cinq ans plus tard, en 661, Ali est assassiné et est enterré dans la terre de Nadjaf. Mu'awiyya se fait proclamer la même année le cinquième calife de l'islam, ce que contestent les partisans d'Ali qui se rallient à l'autorité du fils aîné d'Ali, Hassan (Al-Hassan ibn Ali ; 624-670), petit-fils de Mahomet. Lorsque Hassan meurt en 670, probablement empoisonné, son frère Hussein (Al-Hussein ibn Ali ; 626-680) devient le troisième imam (guide religieux) dans lequel se reconnaissent les partisans d'Ali.

7 Les conflits entre musulmans perdurent. En 680, Hussein quitte La Mecque avec sa famille et un petit groupe de partisans et marche sur l'Irak contre les Omeyyades, pour faire valoir ses droits à la succession califale. Sur la route de Koufa, ville située à 10 kilomètres à l'est de Nadjaf, Hussein et ses partisans sont défaits par les troupes du calife Yazid 1er, le deuxième calife omeyyade qui a succédé à son père Mu'awiya $\mathrm{I}^{\mathrm{er}}$. La tradition rapporte qu'Hussein a été décapité et son corps mutilé à Kerbala²

8 Cette mort tragique d'un descendant direct du prophète, qui fait suite à celle de son père Ali, engendre une rupture définitive au sein de l'islam entre les chiites, les partisans $\mathrm{d}^{\prime} \mathrm{Ali}^{3}$ et les autres musulmans, dits sunnites. Pour les chiites, Ali est un père spirituel, et le seul légataire et successeur de Mahomet. Et seule la filiation de Mahomet, qui n'a pas eu de descendance mâle, peut prétendre légitimement à lui 
succéder. L'importance accordée à Ali tient également au fait qu'il est considéré comme ayant exercé un rôle théologique essentiel au service de la justice et de la vérité. Ali est considéré comme un intercesseur des hommes avec Allah et donc, en quelque sorte, un sauveur.

\section{B. La sépulture d'Ali retrouvée à Nadjaf}

9 Pendant la durée du règne Omeyyade (661-750), les chiites demeurent discrets compte tenu de l'oppression qu'ils subissent puisqu'ils sont traités par les califes de l'islam sunnite comme une secte. Puis, sous les Abbassides, califat sunnite qui commence en 750, la tombe d'Ali est considérée comme redécouverte à Nadjaf. Ce lieu d'inhumation du premier imam chiite devient un sanctuaire, un lieu sacré, et c'est le début d'un pèlerinage dévotionnel.

10 Sous les Abbassides, ce dernier n'est guère apprécié, mais il est toutefois rendu possible notamment grâce à sa sécurisation, car la ville de Nadjaf s'entoure de remparts pour être protégé d'éventuelles attaques. Ensuite, comme l'importance du pèlerinage s'accroît, la ville doit s'étendre pour y accueillir des pèlerins plus nombreux. Des fortifications plus éloignées sont construites et les anciennes sont détruites. Le pèlerinage se poursuit jusqu'à la chute des Abbassides en 1258, vaincus par les Mongols qui contrôlent la Mésopotamie sous l'autorité d'Houlagou Khan (1217-1265), petit-fils de Gengis Khan. Ce nouveau pouvoir risque de mettre en péril le pèlerinage d'autant que les Mongols ont mis à sac nombre de villes irakiennes du califat Abbasside. Mais le nouveau pouvoir non seulement, ne détruit pas Nadjaf, mais semble plutôt respecter le sanctuaire qu'est Nadjaf et participe même à sa protection. Le pèlerinage continue donc pendant plusieurs siècles, jusqu'à une période moins propice au XIV $\mathrm{V}^{\mathrm{e}}$ siècle, lorsque les Turkmènes dominent à leur tour dans la région. Le sanctuaire de l'Imam Ali est alors vandalisé.

\section{L'intensité variable du pèlerinage selon les vicissitudes politiques}

11 La phase politique suivante, sous l'ère ottomane, à compter de 1500 , engendre à nouveau un pèlerinage à éclipses selon le niveau de conflictualité entre les perses et les ottomans. Lorsque ce niveau est faible, et notamment dans les périodes de paix, le pèlerinage est intense, l'aménagement du mausolée d'Ali est amélioré même si les institutions religieuses chiites et nombre d'habitants éprouvent des difficultés à accepter l'emprise ottomane dirigée par le califat sunnite.

$12 \mathrm{Au}$ XIXe siècle, le pèlerinage est périodiquement limité en raison d'attaques venues d'Arabie, donc de sunnites. Par exemple, au début du XIX ${ }^{\mathrm{e}}$ siècle, en 1801 ou 1802, le mausolée d'Ali est endommagé au cours d'un raid mené par des tribus bédouines menées par le chef wahhabite Abdelaziz ben Mohammed ben Saoud. Mais le mausolée, après chaque dépravation, est chaque fois remis en état.

$13 \mathrm{Au}$ début $\mathrm{du} \mathrm{XX}^{\mathrm{e}}$ siècle, une autre période, 1914-1920, n'est guère favorable au pèlerinage: c'est la Première Guerre mondiale, puis Nadjaf, encouragée par ses prêcheurs chiites, se défend, en vain, contre la volonté de conquête des Britanniques. Cette résistance de Nadjaf aux Britanniques explique que la ville soit parfois appelée la « capitale de la révolution » ou le " centre de la révolution ». En 1932, les Britanniques doivent accepter l'indépendance de l'Irak, même s'ils y conservent des bases militaires. 
Ils installent sur le trône le roi Fayçal I ${ }^{\text {er }}$ qui n'était pas forcément le choix des Irakiens. Mais, comme ce roi est favorable à la tolérance religieuse, cela favorise le pèlerinage à Nadjaf qui se poursuit au fil des décennies sous la royauté irakienne.

\section{La république d'Irak, guère favorable au pèlerinage}

14 Le 14 juillet 1958, un coup d'État, mené par le général Kassem, renverse la monarchie hachémite. L'Irak devient une république qui va se révéler fort défavorable au pèlerinage chiite. Effectivement, comme les dirigeants de la république sont pour l'essentiel sunnites, l'attitude de Bagdad vis-à-vis des chiites se durcit, tout particulièrement sous Saddam Hussein ${ }^{4}$ qui organise un régime dictatorial. Les chiites, considérés comme des opposants, subissent des expropriations, des emprisonnements, des condamnations à mort et même l'assassinat de plusieurs chefs religieux. Nadjaf perd de l'influence d'autant que, contrairement aux périodes précédentes, peu d'étrangers viennent bénéficier d'enseignements théologiques à Nadjaf, d'autant que des chefs religieux s'exilent, principalement en Iran, en Syrie et à Londres, pour assurer leur propre sécurité.

Ainsi, pendant les décennies de son pouvoir dictatorial, Saddam Hussein joue à la fois de l'oppression, par des menaces et des meurtres ciblés de grandes figures religieuses chiites $^{5}$, et de l'entrisme en tentant de noyauter le chiisme irakien par l'intrusion d'officiers de sécurité qui se disent étudiants en religion. Il joue aussi sur la rivalité entre les différents ayatollahs pour affaiblir le chiisme. Dans les années 1980, la guerre entre l'Irak et l'Iran rend quasiment impossible la venue à Nadjaf de pèlerins d'Iran et même d'autres pays.

16 À la fin du XXe siècle, le mausolée subit à nouveau de graves dommages pendant la guerre du Golfe de 1991. Des opposants chiites au régime dictatorial s'étant retranchés dans l'enceinte du monument, Saddam Hussein donne l'ordre à la garde républicaine de le prendre d'assaut, provoquant des morts et endommageant le mausolée. Ce dernier est ensuite fermé pendant deux ans, officiellement pour cause de réparations. Puis, durant les dernières années du règne de Saddam, le mausolée est en partie reconstruit par les autorités irakiennes elles-mêmes pour ne pas trop s'aliéner et perdre totalement le contrôle du sud du pays en majorité chiite.

\section{E. Les soubresauts après l'invasion américaine}

Les années qui suivent l'invasion américaine de l'Irak en 2003 voient se desserrer l'étau du régime dictatorial de Saddam Hussein qui avait limité, et même empêche certaines années, Nadjaf d'accueillir des pèlerins. Mais elles n'apportent pas une sécurité totale pour les pèlerins. Le sanctuaire est pris pour cible à de nombreuses reprises. Le 10 avril 2003, le dignitaire chiite Abdul-Majid al-Khoei, considéré comme un chef chiite irakien "modéré », fils du Grand ayatollah Abu al-Qasim al-Khoei, est assassiné à l'arme blanche aux abords du mausolée. Le 24 août de la même année 2003, un attentat à l'explosif vise à Nadjaf le domicile d'un autre haut dignitaire chiite, l'ayatollah Mohammad Saïd Hakim, l'oncle de l'ayatollah Mohammad Baqer al-Hakim, tuant trois personnes de son entourage. Ses proches accusent alors les partisans d'un chef chiite rival, Moqtada al-Sadr, connu pour ses positions radicales sur la présence américaine 
en Irak, d'être à l'origine de cette attaque. Mais ce dernier nie catégoriquement toute implication dans la tentative d'assassinat du chef religieux.

Puis, le 29 août 2003, une voiture piégée explose sur le parvis du mausolée au moment de la prière du vendredi, causant la mort de 82 personnes, dont le Grand ayatollah Mohammed Baqir al-Hakim, chef du conseil suprême islamique irakien, dirigeant notamment l'Assemblée suprême de la révolution islamique en Irak. L'explosion engendre également quelque 230 blessées. L'attentat a été perpétré au moment où les fidèles, rassemblés en masse pour la prière du vendredi, quittaient la mosquée du mausolée d'Ali. La plupart étaient venus écouter le prêche de ce Grand ayatollah, le plus respecté des dignitaires religieux chiites. Ironie du sort, il avait blâmé les partisans de Saddam Hussein pour leurs attaques menées contre les troupes américaines et appelé les chiites à plus de modération. L'attentat visait donc directement, semble-t-il, ce vieil opposant à Saddam Hussein qui a vu une grande partie de sa famille décimée par le régime de l'ancien dictateur. La voiture piégée, une Volkswagen, était en effet placée à l'entrée sud du mausolée, non loin de la porte par laquelle le chef religieux avait pris l'habitude, depuis son retour d'exil en mai 2003, de quitter la mosquée après son prêche du vendredi. C'est alors l'attaque terroriste la plus meurtrière en Irak depuis la chute du régime de Saddam Hussein. "L'ayatollah Mohammad Baqer alHakim est devenu un martyr ", a affirmé depuis Téhéran, des sanglots dans la voix, le neveu de ce haut dignitaire chiite qui a vécu vingt-trois ans en exil dans la capitale iranienne.

L'année suivante, le 24 mai 2004, un tir de mortier atteint le mausolée, causant d'importants dégâts. Le 5 août de la même année, dans un contexte tendu entre les différentes communautés irakiennes, Moqtada al-Sadr et son Armée du Mahdi se retranchent dans la mosquée, d'où ils lancent des attaques contre la police et les forces de la coalition dirigée par les États-Unis. Deux ans plus tard, le 10 août 2006, un terroriste se fait exploser à proximité de la mosquée, tuant 40 personnes et blessant grièvement une cinquantaine d'autres.

Puis, après ces trois années d'insécurité 2003-2006, la ville de Nadjaf se trouve relativement épargnée par la violence ${ }^{6}$ et son contexte géopolitique interne et externe change profondément par rapport à l'époque de Saddam Hussein et évolue plutôt favorablement pour Nadjaf, avec des effets structurants.

\section{F. Une évolution favorable au retour d'un pèlerinage dévotionnel très fréquenté}

21 D'une part, les gouvernements irakiens postérieurs à 2003 ne sont plus, comme le régime de Saddam Hussein, opposés à permettre à Nadjaf d'exercer son rôle de ville de pèlerinage, bien au contraire. D'autre part, les relations de l'Irak avec le pays au monde comptant le plus de chiites, l'Iran, se sont profondément améliorées et la circulation transfrontalière est devenue possible, permettant aux Iraniens d'effectuer leur pèlerinage au mausolée d'Ali. En conséquence, les années postérieures à 2003 voient le début de nouvelles opérations de restauration du mausolée, voire d'embellissement, tout particulièrement grâce à des dons en nature ou financiers en provenance d'Iran. Par exemple, la réfection ou l'ajout de la grande majorité des mosaïques, décorations, ainsi que la façade dorée du tombeau sont réalisées par des artisans iraniens. Des tapis indiquent de façon discrète « don de la ville d'Ispahan » ou « don de la ville de Shirâz ». 

de pèlerins, sans qu'un chiffre précis puisse être proposé. Cet essor du pèlerinage s'entend dans la diversité des langues parlées par les pèlerins et se lit notamment dans les pourtours de la ville, avec de multiples constructions récentes pour héberger les pèlerins, dans le développement de l'aéroport.

Le cœur du pèlerinage dévotionnel est, bien entendu, le mausolée d'Ali, au centre de la vieille ville qui s'étale sur un plateau, même si Nadjaf dispose de nombreux autres sanctuaires. Ce mausolée occupe quatre hectares, en considérant le sanctuaire proprement et la cour qui l'entoure. Son aspect actuel, faisant suite à différents monuments depuis le $\mathrm{X}^{\mathrm{e}}$ siècle, remonte pour l'essentiel au XVI $\mathrm{X}^{\mathrm{e}}$ siècle. Son dôme doré domine le paysage visuel, et forme un repère visible de loin. Pour le rejoindre, il est possible de parcourir les ruelles de la vieille ville, lieux de nombreux magasins où s'alignent de multiples portraits d'Ali, puis une clôture de sécurité.

Document $1:$ Une des nombreuses représentations d'Ali, le quatrième calife de l'islam et le premier imam du chiisme, figurant dans les magasins ou les rues de Nadjaf

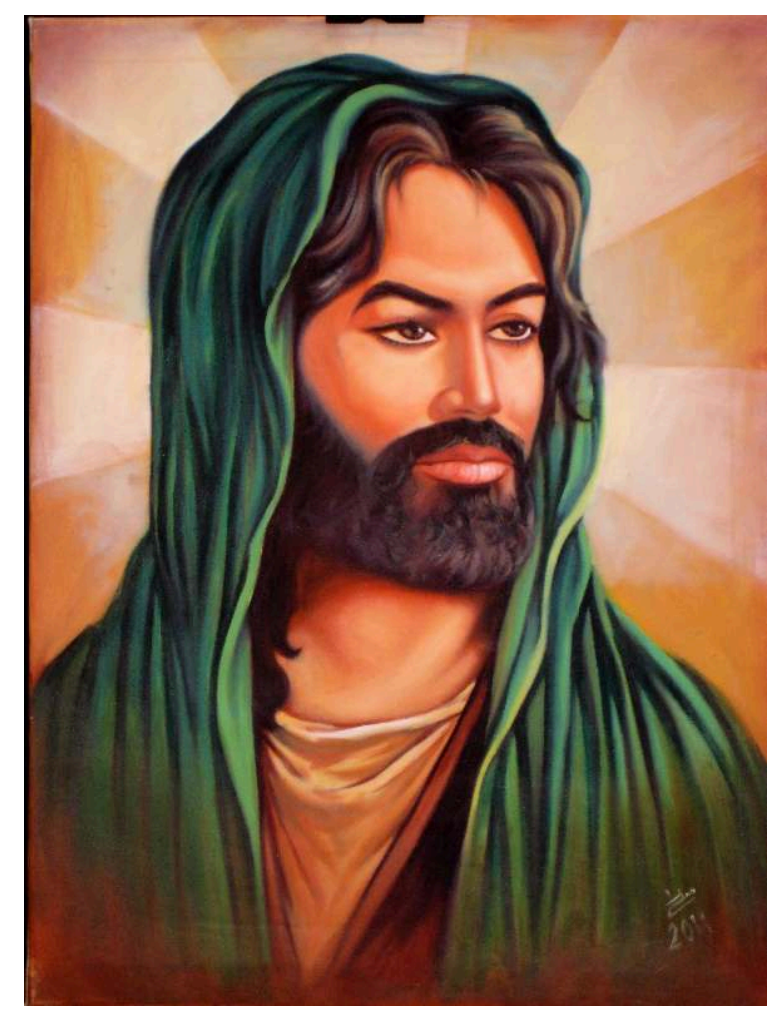

Cliché Gérard-François Dumont - mars 2011. 
Document 2 : La porte Est du mausolée d'Ali à Nadjaf. Au premier plan, des barrières de protection, les pèlerins et visiteurs devant passer par un checkpoint

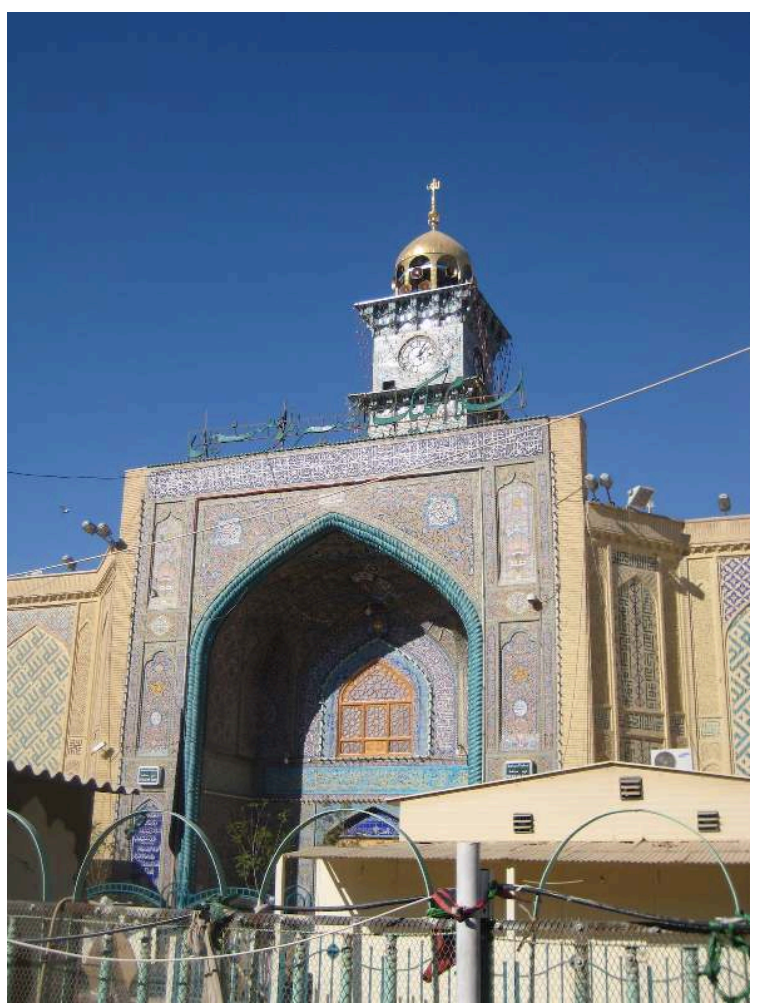

Cliché Gérard-François Dumont - mars 2011.

Document 3: Le plan du centre-ville de Nadjaf tel qu'il est diffusé sur le guide touristique supervisé par le professeur Docteur Abdul-Razzaq Abdul-Jalel Al-Issa. Le mausolée d'Ali, au centre, est entouré de quatre quartiers (Al-Mishraq, Al-Buraq, AlHuweshh et Al-Emarah,) qui permettent de rejoindre le mausolée par des ruelles sauf si l'on parvient par la voie la plus large, à l'ouest du mausolée. La carte indique d'autres sanctuaires et des mosquées, diverses écoles, ainsi que des hôtels et restaurants. 


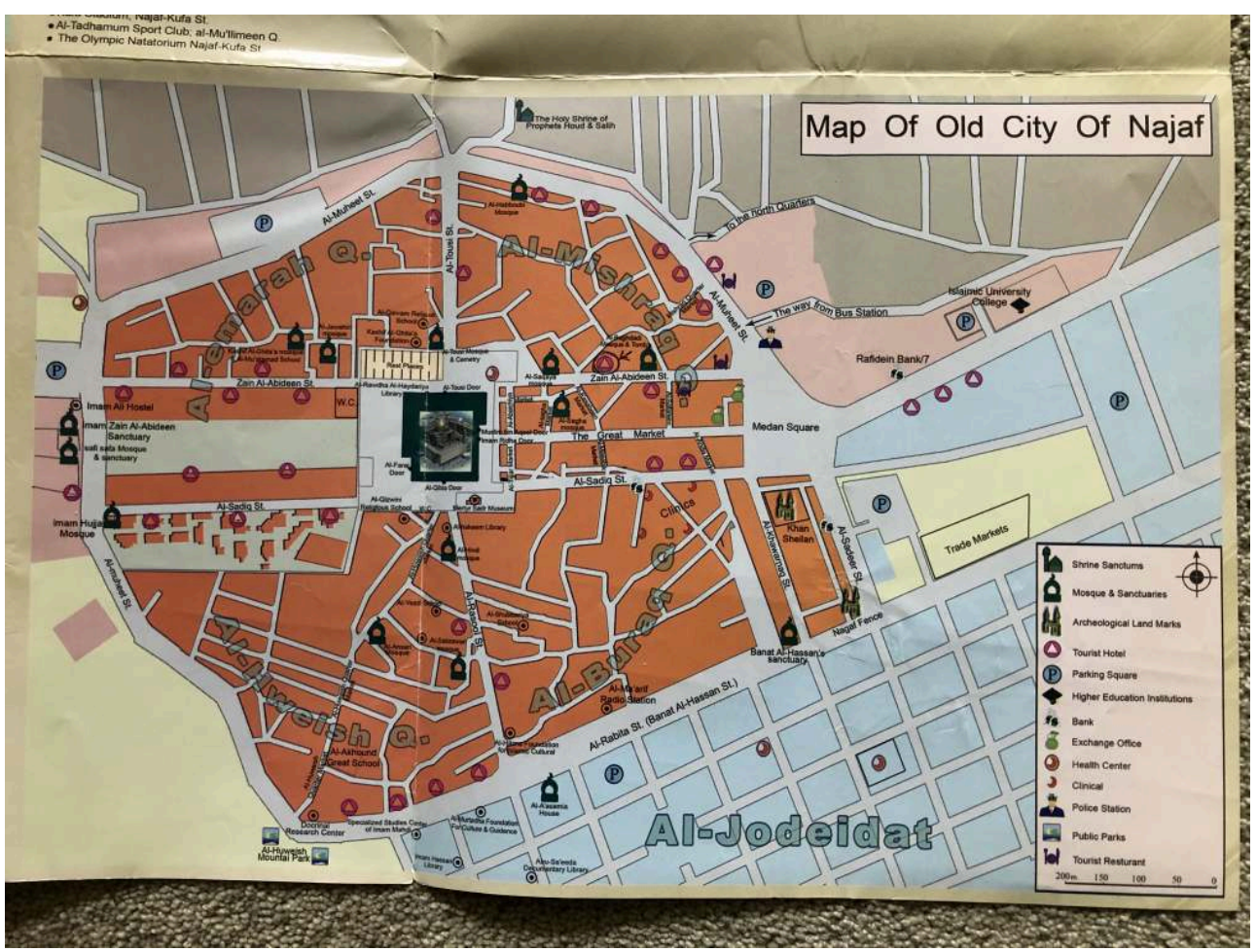

Cliché Gérard-François Dumont - mars 2011

La porte franchie, le pèlerin parvient dans la cour qui entoure le mausolée. Le contraste est saisissant entre la taille du mausolée et la dimension de la cour si l'on arrive par les ruelles du centre-ville. Cette cour est entourée de hauts murs pour assurer la sécurité du mausolée et des pèlerins. Dans cette cour, des groupes de pèlerins assis sur le sol écoutent l'histoire de l'imam Ali telle qu'elle est relatée par les guides depuis des siècles. Au fil du récit, l'émotion monte chez les pèlerins. Puis nombre d'entre eux en viennent aux larmes. On devine une sorte d'émulation entre les guides à celui qui saura le plus émouvoir son groupe de pèlerins.

En conséquence, Nadjaf remplit ses fonctions de haut lieu de pèlerinage dévotionnel. Voir la foule qui se presse d'abord pour passer le checkpoint, puis pour confier, à l'entrée du mausolée, ses chaussures à la consigne et, ensuite, à l'intérieur, pour toucher le mausolée, l'embrasser, ou y frotter un morceau de tissu, est spectaculaire. Sans oublier ces pères qui portent à bout de bras leur enfant ${ }^{7}$ pour qu'il puisse toucher le mausolée.

27 L'importance du pèlerinage dévotionnel à Nadjaf tient aussi à sa fonction de tête de réseau pour d'autres pèlerinages religieux. D'une part, Nadjaf compte à proximité, à une dizaine de kilomètres, la grande mosquée de Koufa, qui fait partie des étapes de tout pèlerinage. Selon les récits de la tradition chiite, cette mosquée est le lieu où l'Imam Ali fut mortellement blessé par un coup d'épée en 661, alors qu'il était en train d'y prier.

28 Nadjaf est aussi le lieu de départ pour le pèlerinage dévotionnel annuel dans la quatrième ville sainte du chiisme, Kerbala, située à $74 \mathrm{~km}$ au nord de Nadjaf et $88 \mathrm{~km}$ au sud de Bagdad, où se trouvent deux importants mausolées séparés de deux cents mètres, celui d'Hussein, fils d'Ali, et de son demi-frère Abbas ${ }^{8}$. En conséquence, la situation de Nadjaf est assez exceptionnelle. En effet, dans nombre de villes de 
pèlerinage, il est possible de se limiter à une journée, éventuellement sans nuitée. En revanche, la pluralité des lieux saints dont la visite est souhaitée et la proximité entre les deux villes saintes de Nadjaf et de Kerbala signifient l'organisation d'un voyage de plusieurs jours, d'autant qu'il faut prévoir le temps passé aux checkpoints, tant à l'intérieur des villes que sur les routes.

29 Ville de pèlerinage dévotionnel, Nadjaf est donc un lieu de voyages qui ne durent généralement que quelques jours. Mais Nadjaf est également un lieu d'instruction théologique, donc de voyages qui peuvent durer jusqu'à trois ans, et que l'on peut désigner comme des " pèlerinages théologiques ».

\section{Les longs « pèlerinages théologiques »}

En effet, dès 1057, une première école religieuse est créée. Au fil des siècles, de nombreuses autres le sont, ce qui porte leur nombre à plus d'une vingtaine en 2020. Ces écoles religieuses forment des étudiants venant parfois d'autres continents à la théologie chiite, ainsi qu'à la littérature, à la philosophie ou à d'autres disciplines. L'ensemble de ces écoles est appelé la hawsa ou territoire de savoir, ce qu'est donc aussi Nadjaf. Ce terme hawsa désigne donc l'ensemble des écoles de la ville, mais peut s'appliquer aussi à une seule école.

\section{A. De multiples écoles et bibliothèques}

$31 \mathrm{Au}$ fil des siècles, les écoles se sont entourées de bibliothèques ouvertes au public, comme la Bibliothèque de l'Ayatollah Al-Hakim. Les bibliothèques sont réparties dans des mosquées, dans des associations et des écoles ou dans les bâtiments privés. Elles contiennent non seulement des centaines de milliers de livres, mais aussi de nombreux livres rares et parfois des manuscrits historiques uniques, soit écrits par les mains de leurs auteurs, soit résultant de prise de notes pendant des audiences de hauts personnages, soit des copies des textes d'auteurs appréciés. 
Document 4 : Une école religieuse (hawza), celle du grand Ayatollah Al-Hakim. Les porteurs de turban noir, accessoire traditionnel dans les pays chiites, se distinguent car ces personnes sont considérées comme descendant de Mahomet, d'où leur titre honorifique de sayyid. Les autres, porteurs de turbans blancs, ne bénéficient pas de ce titre.

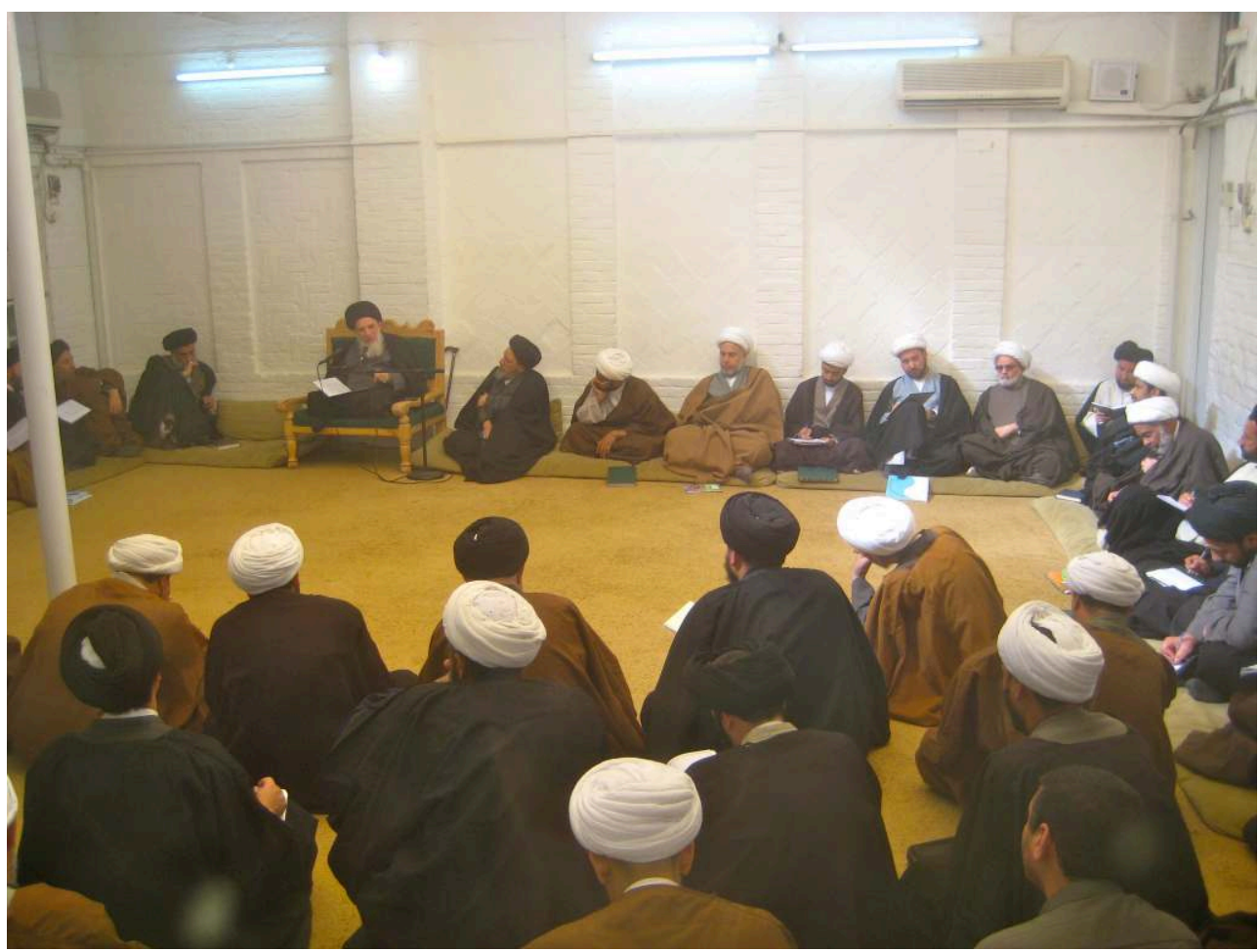

Cliché Gérard-François Dumont - mars 2011

Document 5 : Un des manuscrits rares possédés par la Bibliothèque de l'Ayatollah Al-Hakim

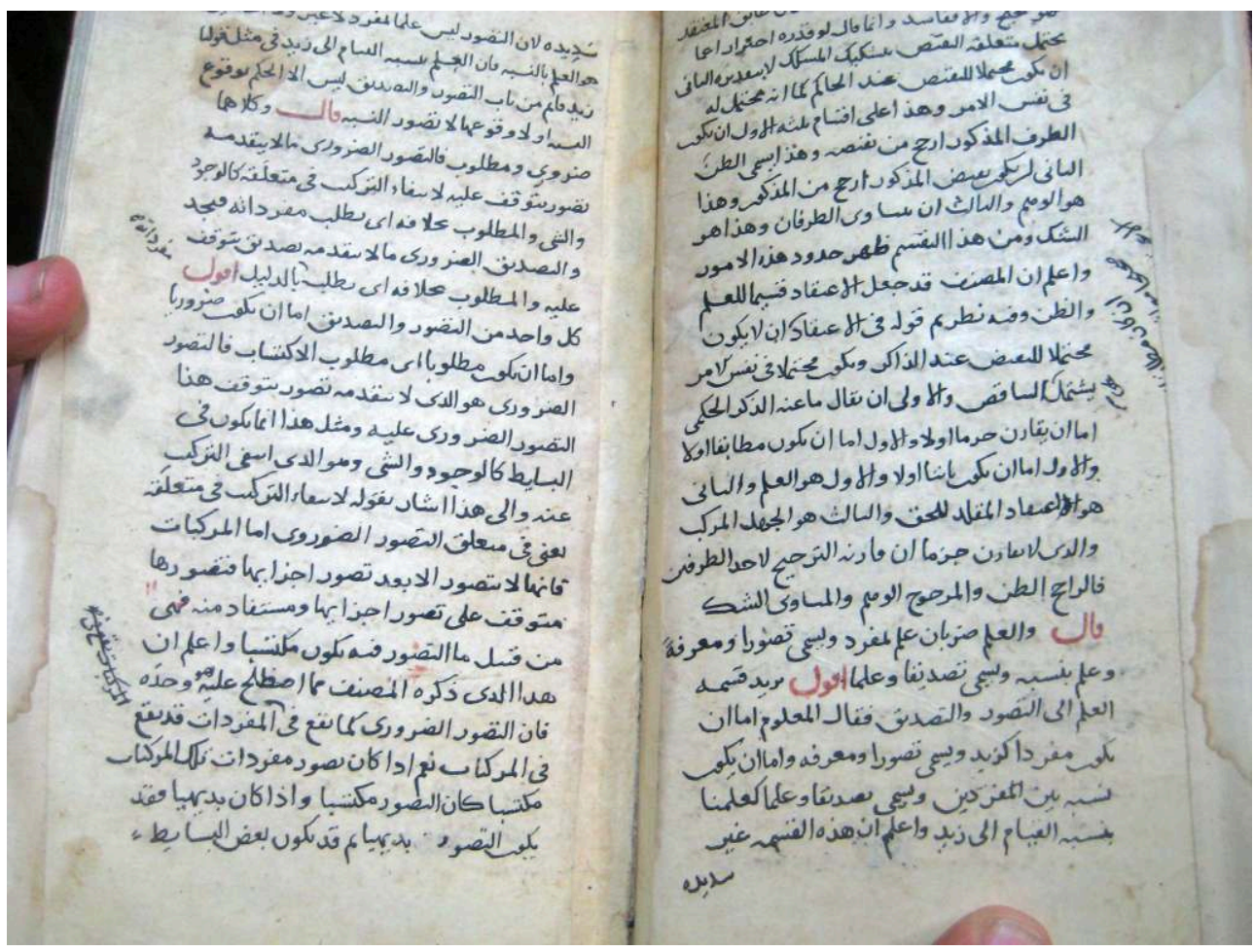

Cliché Gérard-François Dumont - mars 2011 
Les « pèlerinages théologiques » sont donc une composante essentielle de la fonction de Nadjaf comme ville de pèlerinage. Il en résulte, pour satisfaire les enseignements, la présence de nombreux oulémas (théologiens) qui donnent à Nadjaf une place essentielle dans la théologie chiite.

Les écoles religieuses de Nadjaf sont dirigées par des enseignants ayant le titre d'ayatollah, l'un des titres le plus élevé décerné à un membre du clergé chiite', parmi lesquels des grands ayatollahs (ou marja). Pour accéder à un tel titre, des conditions sont requises. D'abord un très grand savoir en matière de juridiction islamique et de capacité à juger. Ensuite, avoir rédigé un traité pratique (Risala Al-Ilmiya) de jurisprudence expliquant ses avis. En outre, il faut bien évidemment faire preuve de piété, de dévotion et de justice. Le marja n'est pas soumis à une élection formelle, mais il est élu de facto dans la mesure où il est choisi par ceux qui le suivent. En effet, la hawza de Nadjaf compte plusieurs ayatollahs et chacun a la liberté de choisir celui qu'il décide de suivre. Les moyens d'une école dépendent donc du nombre de fidèles qui s'en réclament puisque ces derniers participent à son financement par une sorte d'impôt, dont l'importance est essentielle car il permet et a permis, y compris pendant les périodes de répression, d'assurer l'indépendance de la hawza.

Considérant cette dizaine d'ayatollahs qui ont chacun leurs écoles, leurs réseaux et leurs partisans, on pense alors à Rome où les sièges de différents ordres monastiques, des communautés ou de prélatures, tout en professant une foi unique, se trouvent en émulation, voire dans une concurrence qui engendre des effets de synergie favorables à l'attractivité de la ville. À Nadjaf, c'est un phénomène semblable entre les différents ayatollahs.

35 Compte tenu de son attirance religieuse et intellectuelle, Nadjaf connait depuis le milieu des années 2000, une hausse des résidences d'étudiants en religion. Son importance religieuse s'est trouvée reconnue et valorisée par sa désignation, en 2012, comme capitale culturelle du monde de l'islam, avec Dacca et Niamey, par l'Organisation islamique pour l'éducation, les sciences et la culture (Isesco) ${ }^{10}$.

Chaque marja développe, notamment à partir de commentaires des textes coraniques, et en donnant des avis sur l'application des sources islamiques à la période actuelle, une doctrine qui fait référence pour ses disciples. Lorsque, sur tel ou tel point, deux marjas professent une opinion différente, le disciple suit l'opinion de son propre marja, sachant qu'il l'a au départ choisi librement.

\section{B. La théologie dominante à Nadjaf...}

37 La façon dont le marja considère les rapports entre la religion et la politique est un sujet de débat juridique intense et pluriséculaire. À l'origine de l'enseignement religieux à Nadjaf, au Xe siècle, s'instaure une théologie à tendance quiétiste qui s'était d'abord développée à Bagdad dans un contexte de répression des pouvoirs politiques omeyyade et abbasside et de refus d'être politisé. En 1056, les imams doivent quitter Bagdad pour se réfugier à Nadjaf où ils professent notamment le refus de toute fonction politique. La mission qu'ils s'assignent est la transmission du savoir religieux, non l'implication dans des fonctions temporelles.

38 Aujourd'hui encore, selon les écoles dominantes à Nadjaf, que l'on peut appeler « traditionalistes », le religieux ne doit donc pas exercer le pouvoir politique, même s'il 
peut faire valoir son droit de regard sur la politique. En effet, il doit encourager les dirigeants politiques à respecter des principes moraux favorables au bien commun de la population. Par exemple, en 1906, lors de la révolution constitutionnelle iranienne, les responsables religieux de Nadjaf ont émis des fatwas soutenant ce mouvement démocratique.

39 Seul le douzième imam caché, le Mahdi, pourra, à son retour, être à la fois chef religieux et politique. Pendant l'occultation du Mahdi, le pouvoir doit revenir au peuple, à la nation par le biais d'élections démocratiques. Les écoles dominantes à Nadjaf sont donc partisanes d'un régime démocratique garant de la liberté, de la justice et de l'égalité des citoyens.

Cette interprétation traditionaliste dominante à Nadjaf a rayonné différemment selon les périodes, d'autant que l'importance des «pèlerinages théologiques » à Nadjaf a subi les vicissitudes des évolutions géopolitiques. En particulier, son importance s'est réduite dans les deux dernières décennies $\mathrm{du} \mathrm{XX}^{\mathrm{e}}$ siècle sous trois effets. Il y a d'abord eu les actions, souvent violentes, de Saddam Hussein pour réduire l'attractivité religieuse de Nadjaf. Ensuite, la guerre entre l'Irak et l'Iran (1980-1988) a instauré l'impossibilité pour des Iraniens d'effectuer un " pèlerinage théologique » à Nadjaf et a rendu difficile la venue de chiites non iraniens. Enfin, après la révolution iranienne de 1979, la ville sainte de Qom, en Iran, fort soutenue par le nouveau régime de la révolution islamique, s'est trouvée le principal lieu de pèlerinages théologiques chiites, développant principalement une théologie non quiétiste comme précisé ci-après. Toutefois, depuis 2006, après le déclin subi sous le régime de Saddam Hussein, Nadjaf est redevenue, avec Qom en Iran, la principale hawza du monde chiite.

\section{C... différente de la théologie " rationaliste »}

41 Dans leur majorité, les écoles de Nadjaf se positionnent différemment de l'autre interprétation théologique du chiisme, dite " rationaliste ", qui considère que les chefs religieux doivent s'emparer du politique. Selon cette seconde interprétation, il faut donc instaurer un gouvernement islamique. Il ne faut pas attendre le retour du Mahdi pour l'exercice par les religieux des responsabilités politiques les plus élevées. Cette interprétation s'est déployée en Perse en 1501 lorsque le shah Ismaïl, lors de l'instauration de la dynastie des Safavides, a affirmé qu'il était dépositaire des pouvoirs du Mahdi qu'il aurait rencontré dans une caverne. Ainsi, les tenants du Velayat-e faqih, ce qui signifie les conservateurs de la jurisprudence ou encore "gouvernement du docte ", comme Rouhollah Mousavi Khomeini (1902-1989), souhaitent que les marjas gouvernent.

Plus récemment, cette approche a en effet été remise en avant par l'imam Khomeiny qui, exilé entre 1964 et 1978 à Nadjaf, y rédige un manifeste réaffirmant le principe du Velayat-e faqih, qui confère aux religieux la primauté sur le pouvoir politique, manifeste qu'il va mettre en œuvre en Iran à compter de 1979. Après 2003, la république islamique d'Iran a cherché à implanter à Nadjaf son modèle et ses doctrines par une forte présence: financement de la réfection, de l'entretien et de l'agrandissement des mausolées des imams, présence de commerçants, d'entreprises, de banques, de main-d'œuvre iraniennes... Mais, en dépit de ses importants moyens, la république islamique d'Iran n'est pas parvenue à dominer théologiquement Nadjaf et Nadjaf a continué à affirmer sa théologie traditionaliste. 


\section{La spécificité théologique des pèlerinages à Nadjaf attesté par I'histoire récente}

43 Les différences entre les deux théologies, traditionaliste et rationaliste, sont incontestables, par exemple en comparant les discours du guide suprême de la république islamique d'Iran et ceux du plus prestigieux marja de Nadjaf, le grand Ayatollah Ali Sistani.

Elles ont à nouveau été soulignées dans les nombreuses manifestations d'Irakiens, de confession chiite pour la très grande majorité ${ }^{11}$, qui ont ponctué la vie politique irakienne depuis le $1^{\mathrm{er}}$ octobre 2019. Ces manifestations dénonçaient la corruption et le népotisme en Irak et demandaient la démission du Premier ministre irakien Adel Abdel-Mahdi, puis, l'ayant obtenu, notamment avec le soutien du grand ayatollah Sistani, refusaient le choix d'un Premier ministre désigné Mohammed Taoufiq Allaoui, ce dernier ayant participé au régime installé depuis 2003. L'importance de la religiosité chiite à Nadjaf et en Irak a été mise en évidence par le fait que les manifestations ont été suspendues pendant la grande marche d'Arbaïn (ce qui signifie le quarantième), qui part chaque année notamment du mausolée de l'imam Ali pour l'autre Ville sainte de Kerbala et qui marque la fin des quarante jours de deuil après l'anniversaire de la mort de l'imam Hussein.

Hors de cette suspension, les manifestations à Nadjaf et dans les autres villes du sud de l'Irak, ont, directement ou indirectement, témoigné de leur refus de l'interprétation rationaliste du chiisme en s'en prenant à l'Iran, malgré l'importance de la répression (environ 500 morts en Irak dont un certain nombre à Nadjaf) et la confrontation avec des manifestants pro-iraniens. La république islamique d'Iran s'est trouvée accusée d'avoir pénétré les rouages de l'État irakien à tous les niveaux, de contribuer à une corruption généralisée, et d'étouffer l'économie irakienne en prétendant la soutenir. Les manifestants, dont de nombreux chiites venus effectuer un "pèlerinage théologique » à Nadjaf, ont déclamé : «Iran dehors, Irak libre ». Puis, face à l'absence de réformes et à l'ampleur de la répression, le mouvement de contestation s'est durci. Le 27 novembre 2019 à Nadjaf, les manifestants ont incendié une partie du consulat iranien et un couvre-feu a été imposé dans la ville. La répression a été violente, ce qui n'a pas empêché les Irakiens de Nadjaf de débaptiser la rue de l'Imam Khomeiny pour l'appeler « rue de la Révolution d'octobre».

46 Mais, à Nadjaf comme à Bagdad, dans tout le sud irakien, ces manifestants se sont vus opposer des contre-manifestants soutenus par la république islamique d'Iran, dont les partisans du leader chiite Moqtada Sadr qui, après avoir changé de camp au fil des semaines, distingue ses partisans par des "casquettes bleues" comme signe de ralliement. Difficile de dire si les morts et les blessés de Nadjaf sont dus aux contremanifestants ou aux policiers. Dans tous les cas, les seconds sont accusés ou de violence ou de ne pas avoir capable de les empêcher.

47 Le refus de toute référence à la république islamique d'Iran, y compris dans la dimension théologique, s'est même manifesté de façon inattendue le 17 février 2020. Pour la première fois dans l'histoire de la ville, des Irakiennes ont défilé sans les hommes, mais avec les mêmes slogans que ceux des précédentes manifestations, soit "Non à l'Amérique ", "Non à l'Iran». Les manifestantes voulaient dénoncer la mauvaise gouvernance du pays, gangrené par une corruption fort élevée, deux 
puissances, États-Unis et l'Iran, qui soutiennent les gouvernements irakiens, ainsi que le chef chiite Moqtada Sadr qui a récemment retiré son soutien aux manifestants contre le gouvernement et qui semble d'ailleurs avoir perdu des militants à cette occasion.

Les événements du dernier trimestre 2019 et de début 2020 à Nadjaf témoignent donc à nouveau de la spécificité théologique des pèlerinages ayant pour objet d'effectuer des études religieuses dans cette ville, spécificité datant d'un millénaire et qui a résisté aux évolutions géopolitiques de la religion musulmane.

L'attractivité de Nadjaf tient à un troisième type de pèlerinage, post mortem.

\section{Le plus important pèlerinage post mortem}

La raison de ce pèlerinage post mortem tient à ce que tout chiite, quel que soit le pays où il a réellement vécu, souhaite se faire enterrer près d'Ali, donc à Nadjaf ${ }^{12}$. Il en résulte un immense cimetière international, Wadi al-Salam, la "Vallée de la Paix », sans doute le plus grand cimetière du monde et, pour le moins, le plus grand cimetière musulman au monde. En effet, cette véritable "cité des morts", s'étend sur une surface considérable, soit plus de $6 \mathrm{~km}^{2}$. Une sorte de ville nécropole! L'étendue de ce vaste cimetière explique d'ailleurs l'absence de développement de l'urbanisation au nord et au nord-ouest de la ville. Nadjaf est organisée pour que les déplacements liés aux funérailles, des dizaines chaque jour, parfois plus d'une centaine, puissent s'effectuer. Dans ce dessein, des centaines d'entrepreneurs de pompes funèbres, dans leurs échoppes, offrent leur service près du cimetière, là où se trouvent également de petites échoppes qui vendent des biscuits secs ou des boissons rafraîchissantes.

51 Selon les estimations, ce cimetière abriterait entre 5 et 15 millions de tombes... « Dieu seul le sait », comme le dit un vieux fossoyeur. La loi de l'offre et de la demande fait que les concessions se négocient à des prix fort élevés. On dit que certains pèlerins âgés souhaitent décéder au cours de leur pèlerinage au sanctuaire de l'Imam Ali dans l'espoir que la présence d'une grande figure religieuse à leurs côtés permette d'atténuer les souffrances de la mort et d'intercéder en leur faveur.

La fonction et l'organisation du cimetière se sont trouvées à nouveau mises en évidence lors des combats de l'armée et de milices irakiennes contre l'organisation état islamique en 2015-2017. Les dépouilles des combattants chiites tués dans l'ouest du pays lors de cette reconquête du territoire irakien ont été transférées à Nadjaf pour y être enterrées. Et le cimetière illustre la diversité des milices ayant participé à ce combat. En effet, chacune s'est vue affecter un secteur dédié où elle a enterré ses morts. 


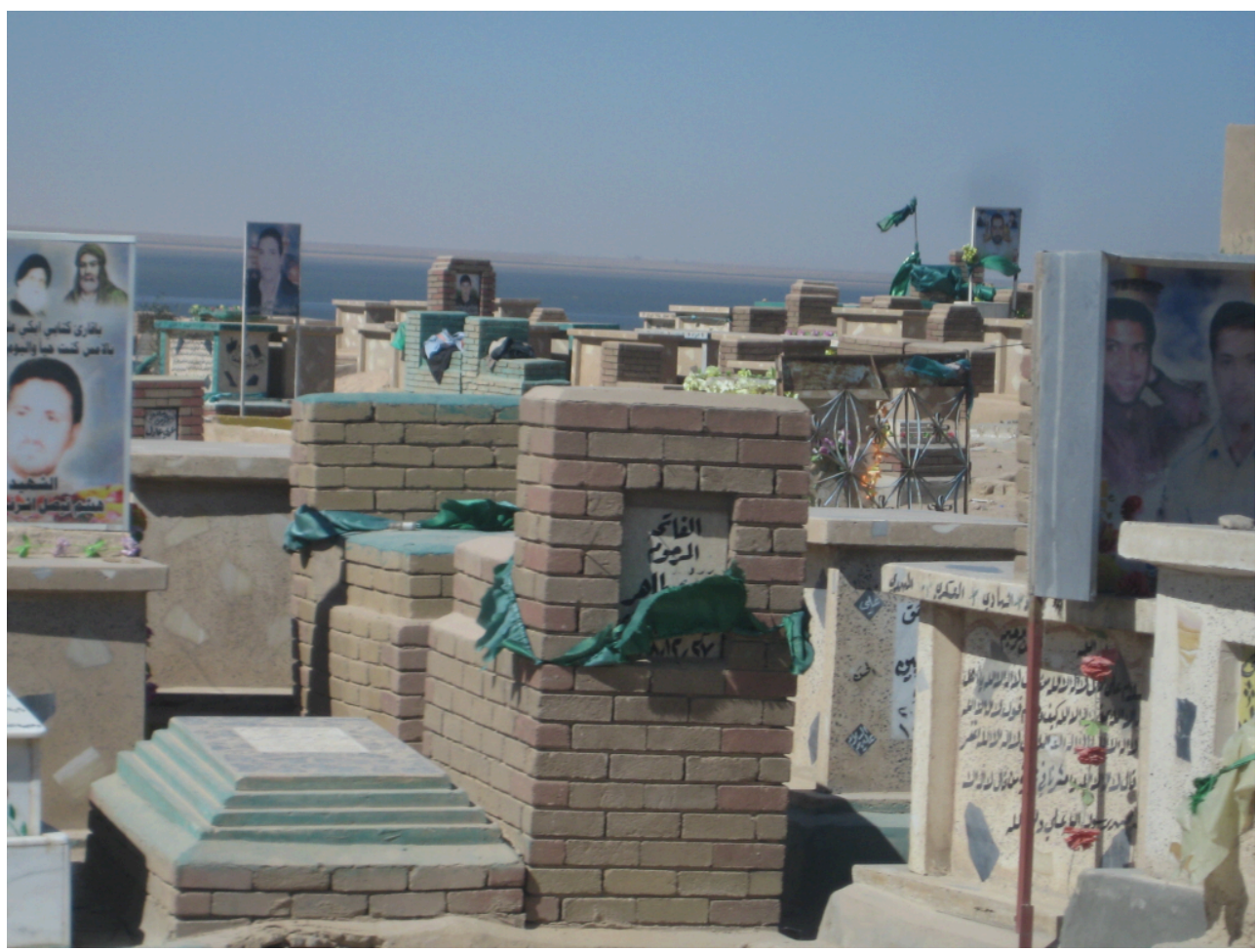

Cliché Gérard-François Dumont - mars 2011.

53 Le cimetière reçoit chaque année la visite de centaines de milliers de pèlerins, venus honorer leurs ascendants ou les prophètes et les grands mystiques qui y sont enterrés. En parcourant la longue route qui le traverse, on aperçoit des tombes ocres à perte de vue dont les plus anciennes remonteraient à plus d'un millénaire, des dômes turquoise et des petits mausolées, dont on peut se rapprocher par des allées poussiéreuses.

L'importance du pèlerinage dévotionnel à Nadjaf est donc due à la centralité spirituelle de cette ville où se trouve le mausolée d'Ali, quatrième calife de l'islam et premier imam du chiisme. Nadjaf est donc une ville sainte comme d'autres dans le monde. Mais elle a des caractéristiques particulières très anciennes qui démultiplient son attraction depuis des siècles. Il s'agit d'une part de l'importance de ses écoles religieuses (Hawza), s'accompagnant d'une production continue de savoir, et attirant des étudiants chiites du monde entier qui y effectuent des " pèlerinages théologiques». S'ajoute d'autre part l'importance de son cimetière, lieu de sépulture souhaité par les chiites du monde entier.

Certes, au fil des siècles, Nadjaf n'a pas toujours pu exercer ces trois fonctions de pèlerinage avec un maximum d'intensité, compte tenu des changements de son contexte géopolitique qu'elle a subis et du poids relatif variable de ceux qui adhèrent à une autre théologie chiite que la sienne, qui est traditionaliste. Toutefois, Nadjaf pourrait, dans un cadre local et national attractif mieux organisé et mieux gouverné, être aussi une ville très prisée pour des touristes intéressés par son patrimoine, d'autant que, contrairement à La Mecque, la ville n'est pas fermée aux non-musulmans. 


\section{NOTES}

1. Ce texte ne comporte pas d'éléments bibliographiques. En effet, il résulte d'une étude de terrain et des conversations avec des Irakiens, des autorités religieuses de Nadjaf et de membres des diasporas moyen-orientales en Europe. Pourtant, à Nadjaf, les sources écrites, rédigées au fil des siècles en arabe, sont très nombreuses. Lorsque j'ai demandé à l'une des quatre plus hautes autorités religieuses de Nadjaf pourquoi certains des textes n'étaient pas traduits, ce qui permettrait notamment de faire connaître en Occident une autre théologie chiite que celle de la République islamique d'Iran depuis Khomeiny, il m'a été répondu la crainte de traductions inappropriées. Une réponse qui masque la complexité des relations de Nadjaf avec la République islamique d'Iran.

2. Où a été construit son mausolée, quatrième lieu saint des Chiites. Hussein est considéré par les chiites comme un héros révolutionnaire qui a tenté de renverser un système politique qui a trahi l'idéal coranique.

3. Ali est donc à la fois le quatrième calife «légitime " et le premier imam pour les chiites, premier d'une chaîne de douze imams qui se termine avec Mohamed al-Mahdi al-Qaim en 940, d'où le nom de chiisme duodécimain. Comme Mahdi, dans un contexte où les sunnites voulaient éliminer les chiites, vivait dans la clandestinité et donc ne se montrait pas, il est considéré par les chiites comme occulté. Mais, selon la théologie chiite, ce douzième calife reviendra à un moment indéterminé pour établir un règne de paix et de justice. Ce sera la fin de son occultation majeure.

4. Vice-président (1968-1979) puis président (1979-2003) de la République Irakienne, suite au renoncement " précipité » de son prédécesseur, officiellement pour « raison de santé ».

5. Comme l'assassinat de Mohammad Sâdiq as-Sadr, père de Moqtada al-Sadr, en 1999.

6. Ce qui n'empêche pas le maintien de multiples checkpoint.

7. Il en est sans doute de même du côté des femmes, sachant que, après une dernière fouille, les hommes et les femmes se séparent, une partie du sanctuaire leur étant à chacun réservée.

8. Mort également lors de la bataille de Karbala en 680 .

9. En effet, le clergé chiite, dont l'existence est une autre (MANQUE DU TEXTE ?) distingue entre le chiisme et le sunnisme, est très hiérarchisé.

10. Dont le siège permanent se trouve à Madinat $\mathrm{Al}$ Irfane, dans le quartier Hay Riyad de Rabat, donc à proximité du pôle qui regroupe les universités et les écoles les plus importantes de la capitale marocaine.

11. Les Irakiens sunnites n'ont guère été présents dans les manifestations qui opposaient des irakiens chiites à un gouvernement au sein duquel le pouvoir est essentiellement détenu par des irakiens chiites.

12. Ce souhait fait penser à celui des membres de la confrérie musulmane des mourides qui souhaitent se faire enterrer à Touba, leur ville sainte au Sénégal.

\section{RÉSUMÉS}

Nadjaf, ville située à $160 \mathrm{~km}$ au sud de la capitale irakienne Bagdad, tient une place très importante comme ville de pèlerinage et lieu de départ de la principale procession chiite annuelle. En outre, sa fonction de pèlerinage dévotionnel se complète doublement, d'une part, 
par ce qu'il convient d'appeler de longs "pèlerinages théologiques » et, d'autre part, par un dernier pèlerinage post mortem de la dépouille des croyants.

Najaf, a city located $160 \mathrm{~km}$ south of the Iraqi capital Baghdad, plays a very important role as a pilgrimage city and the starting point of the main annual Shiite procession. In addition, its function as a devotional pilgrimage is complemented doubly, on the one hand, by what is known as long "theological pilgrimages" and, on the other hand, by a final post-mortem pilgrimage of the remains of believers.

INDEX

Keywords : Middle East, Iraq, Nadjaf, Karbala, Islam, Shia, Ali, Theology, Pilgrimage

Mots-clés : Moyen-Orient, Irak, Nadjaf, Kerbala, Islam, Chiisme, Ali, Théologie, Pèlerinage

\section{AUTEUR}

\section{GÉRARD-FRANÇOIS DUMONT}

Sorbonne Université

Population \& Avenir

35 Avenue Mac Mahon 75017 Paris

gerard-francois.dumont@wanadoo.fr 